\title{
Non Invasive Assessment of Hepatic Fibrosis and Necro-inflammatory Activity in Pediatric Patients with Chronic Hepatitis C Virus Infection: A Pilot Study of the Role of FibroTest and ActiTest in Children Mortada El-Shabrawi*, Nabil Abd El-Aziz", Hany Riad", Hussin Mahdy",
M.Mostafa AbdEl-Ghafar*, Mai Sherif**, Ahmed El-Hennawy****
}

\begin{abstract}
Hepatitis $\mathrm{C}$ virus (HCV) is a major cause of chronic hepatitis and hepatic fibrosis. The overall sero-prevalence in Egyptian children in two cross sectional studies from Southern and Northern Egypt were $\mathbf{8 . 7 \%}$ and $\mathbf{2 4 . 3 \%}$ respectively. Finding the best method to evaluate and manage children infected with HCV continues to be a challenge. Liver biopsy, due to its limitations and risks, is an imperfect gold standard for assessing the severity of the most frequent chronic liver diseases. Noninvasive alternatives to liver biopsy in patients infected with HCV include 2 combinations of simple serum biochemical markers: FibroTest (FT) for the assessment of fibrosis, and ActiTest (AT) for the assessment of necro-inflammatory activity. Although, the uses of FT and AT have been validated in adults with chronic HCV infection and is probably of great benefit, yet, there is lack of relevant data concerning their role in pediatric patients. In this pilot study, we attempted to verify the possibility of implementation of FT and AT as non invasive markers in assessment of the degree of hepatic fibrosis and necro-inflammatory activity in pediatric patients with chronic HCV infection in comparison to liver biopsy. Fifty patients, aged 2.0-18.0 years (with a mean of $10.52 \pm 4.83$ years), with chronic $\mathrm{HCV}$ infection were studied. Two assessments have been done, within 24-hour-duration, one of a liver biopsy specimen and the other with FT and AT measured in serum sample. A highly significant linear trend between FT related fibrosis and fibrosis stage by histopathological examination was found ( $p$ value $<0.0005$ ). A highly significant correlation was also found between both parameters $(r=0.811)$. As well, a highly significant correlation was found between AT and necro-inflammatory histological activity index (HAI) with $r=0.591$ and $p<0.0005$. FT and AT are potential non-invasive methods for assessment of hepatic fibrosis and necro-inflammatory activity in pediatric patients with chronic $\mathrm{HCV}$ infection.
\end{abstract}

Introduction:

Hepatitis C virus (HCV) infection is a major cause of chronic hepatitis and hepatic fibrosis (Paik, 2008). Egypt has the highest prevalence of HCV infection in the world, averaging $12-24 \%$ in the general population. HCV Genotype 4 is the prevailing genotype in Egypt (90\%) (El-Hawary et al., 2007). The overall sero-prevalence in Egyptian children in two cross sectional studies from Southern (upper) Egypt (Medhat et al. 2002), and Northern (Lower Egypt) (Habib et $\boldsymbol{a l}$., 2001) were $8.7 \%$ and $24.3 \%$ respectively.In developed countries, incidence of transfusion-related $\mathrm{HCV}$ infection has markedly decreased, and perinatal transmission has become the most important route of viral spread in children (Jonas, 2002). However, in developing countries, unsafe blood transfusions and invasive surgical procedures are still a major route of viral transmission (Wasley and Alter 2000). Worldwide, the major clinical consequence of chronic hepatitis $\mathrm{C}$ infection is the progression to cirrhosis and its potential complications: hemorrhage, hepatic insufficiency, and primary liver cancer (ElHawary et al., 2007).

*Pediatric Department Ahmed maher teaching Hsopital

**Clinical Pathology Department and ***Pathology Department, Faculty of Medicine, Cairo University.
Cirrhosis of the liver is characterized by a profound derangement of liver architecture caused by fibrosis and nodule formation (Bosch, 2009). The current understanding of HCV infection has been advanced by the concept of liver fibrosis progression (El-Hawary et al., 2007).

In pediatric patients, the outcome of $\mathrm{HCV}$ infection is uncertain as a result of the variation of clinical course of infection (Habib et al., 2001). Children with chronic hepatitis $\mathrm{C}$ will be at risk for progressive liver disease as they age (Goodman et al., 2008). Little is known about the risk factors for the progression of liver disease in children, and few well-designed pediatric clinical trials have been conducted. Therefore, the decision to treat (or not) children with this viral pathogen is currently a contentious endeavor (Jonas, 2002).

Finding the best method to evaluate and manage patients infected with HCV continues to be a challenge (Ngo et al., 2006). Liver biopsy, due to its limitations and risks, is an imperfect gold standard for assessing the severity of the most frequent chronic liver diseases (Poynard et al., 2008). It is an invasive procedure, unpleasant for the patient, expensive, not free from severe complications, and prone to sampling errors due to the very small size of needle biopsy specimens (Bosch, 2009). It is thus no 


\section{Mortada El-Shabrawi, et al}

longer considered mandatory as the first-line indicator of liver injury (Poynard et al., 2008).

Noninvasive alternatives to liver biopsy in patients infected with $\mathrm{HCV}$ include 2 combinations of simple serum biochemical markers: FibroTest (FT) (Biopredictive) for the assessment of fibrosis, and ActiTest (AT) (Biopredictive) for the assessment of necro-inflammatory activity. They can be used as an alternative to liver biopsy for determining the severity of chronic HCV infection (Ngo et al., 2006).

FT has prognostic values at least similar to that of liver biopsy, with the advantage of being noninvasive (Ngo et al., 2006). Therefore, it appears to be a very valuable method for detecting early stages of fibrosis among patients with chronic HCV infection, allowing avoiding the progression of liver damage and end-stage liver disease. It is easy to perform and therefore allows regular follow-up of the course of liver fibrosis (Dolmazashvili et al., 2008). Not only, FT could be used as an alternative to liver biopsy in chronic HCV (Poynard et al., 2007), but also in the three other common fibrotic liver diseases: (Poynard et al., 2005) namely chronic hepatitis B virus (HBV) infection (Myers et al., 2003), alcoholic liver disease (ALD) (Naveau et al., 2005) and non-alcoholic fatty liver disease (NAFLD)( Ratziu et al., 2006). A recent overview by French health authorities officially approved non invasive biomarkers such as FT and AT as first line estimates of fibrosis in patients with chronic hepatitis $\mathrm{C}$, recommended reimbursement by social security and approved liver biopsy only as second line estimate in case of discordance or non interpretability of non invasive marker (Poynard et al., 2007).

Although, the use of FT and AT have been validated in adults and proved great benefit, yet, there is lack of relevant data concerning their role in pediatric patients. In this pilot study, we aimed to verify the possibility of implementation of FT and AT as non invasive markers in assessment of the degree of hepatic fibrosis and necroinflammatory activity in pediatric patients with chronic $\mathrm{HCV}$ infection in comparison to the gold standard: liver biopsy.

\section{Patients and Methods: \\ Patients:}

Study group included 50 pediatric patients with chronic HCV infection recruited from Hepatology Unit of Cairo University Children Hospital, the largest tertiary care Pediatric Hospital in Egypt, from May 2007 to June 2008. Patients' ages ranged from 2.0 to 18.0 years (with a mean of $10.52 \pm 4.83$ years). Fifteen were female $(30 \%)$ and 35 were male (70\%). Thorough history and physical examination were performed. Laboratory investigations included: total and direct serum bilirubin, hemoglobin level, reticulocytic count and biochemical tests of liver function). Chronic HCV infection was diagnosed by detection of anti-HCV antibodies and confirmed by qualitative and quantitative $\mathrm{HCV}$ ribonucleic acid (RNA) detection by the polymerase chain reaction (PCR). Patients were diagnosed and their grading and staging according to laboratory and biopsy results were the basis for their further management. All procedures were in accordance with the current revision of the Helsinki Declaration, and all participants gave informed consents (Rothman. 2000).

\section{Exclusion criteria:}

Patients/guardians who refused to consent for liver biopsy were excluded from the study. Patients with acute hepatitis, acute hemolysis, chronic hemolysis, Gilbert's disease, or with associated extra-hepatic illnesses that may affect the results of FT and AT were also excluded from the study.

\section{Inclusion criteria:}

For inclusion in the study, a successful assessment of liver pathology was needed to be done within $24 \mathrm{~h}$ by both:

1. Histopathological examination of a percutaneous liver biopsy specimen.

2.FT and AT measured in a serum sample.

\section{Biochemical markers:}

FibroTest and ActiTest (Biopredictive, Paris, France) were performed according to published recommendations (Poynard., 2007). FT and AT are noninvasive blood tests that combine the quantitative results of 6 serum biochemical markers, [ $\alpha 2$-macroglobulin, haptoglobin, glutamyl transpeptidase (GGT), total bilirubin, apolipoprotein A1, and alanine aminotransferase (ALT)] with patient age and sex data in a patented artificial intelligence algorithm (Poynard., 2003), to generate a measure of fibrosis and necro-inflammatory activity in the liver (Myers et al., 2003, Thabut et al.,2003, Callewaert et al., 2004, Castéra et al., 2005 and Halfon et al., 2006). This method provides a numerical quantitative estimate of liver fibrosis ranging from 0.00 to 1.00 and grades $\mathrm{A} 0-\mathrm{A} 3$ of the well-established METAVIR scoring system, which grades fibrosis from F0 (no fibrosis) to F4 (cirrhosis). An algorithm has been suggested that would classify patients into 3 groups: no or minimal fibrosis (FT 0-0.31), moderate fibrosis (FT 0.31-0.58), and severe fibrosis (FT 0.58-1.00) (Poynard et al., 2004).

\section{Methods:}

We measured GGT, ALT, total bilirubin, apolipoprotein A1, and haptoglobin with a Cobas Integra 400 analyzer and Roche Diagnostics reagents. $\alpha 2$-macroglobulin was assayed with a Cobas Integra 400 Turbidimetry but with Dako utility channel reagents. All tests were performed by personnel blinded to all patient characteristics, including biopsy results.

\section{Histopathological study:}

Liver biopsy was performed in all patients as indicated during the management of this cohort of chronic HCV infected children; not related to the study protocol. Liver biopsies were all standard percutaneous samples, and the threshold of adequacy for histological assessment was the 


\section{Non Invasive Assessment of Hepatic Fibrosis......}

presence of more than five portal tracts. A single experienced pathologist, who was unaware of the biochemical markers, evaluated the stage of fibrosis and grade of necro-inflammatory activity according to the METAVIR scoring system (Bedossa and Poynard 1996). Fibrosis was staged on a scale of $0-4$ : where F0, no fibrosis; F1, portal fibrosis without septa; F2, few septa; F3, numerous septa without cirrhosis and F4, cirrhosis.

\section{Statistical analysis:}

Data were summarized as median and inter-quartile range (25th-75th percentiles) and displayed as box and whisker plots. Receiver Operating Characteristic (ROC) curves was used to study the diagnostic performance of FT and AT and select optimum cutoff values. SPSS 15.0 ( ) was used for statistical treatment of data (SPSS Inc., Chicago, Ill).

\section{Results:}

Fifty patients aged 2.0-18.0 years (with a mean of $10.52 \pm 4.83$ years), diagnosed as chronic HCV were included in the study. By histopathological examination of liver biopsy; $20(40.0 \%)$ of them had no fibrosis, 4 $(8.0 \%)$ minimal fibrosis, $19(38.0 \%)$ mild fibrosis, 1 $(2.0 \%)$ moderate fibrosis and $6(12.0 \%)$ had severe fibrosis.

As demonstrated in Fig. 1, the median $\left(25^{\text {th }}-75^{\text {th }}\right.$ percentile) FT values increased linearly with advancing hepatic fibrosis stage. FT values were $0.16(0.07-0.25)$ in patients with no fibrosis, $0.19(0.18-0.24)$ in minimal fibrosis, $0.41(0.20-0.66)$ in mild fibrosis, 0.54 in the single moderate fibrosis-patient and finally $0.66(0.43-$ 0.77 ) in cases of severe fibrosis. This linear trend was highly significant with $\mathrm{p}<0.0005$. A highly significant correlation was also found between individual FT values and the respective fibrosis stage, $r=0.811, \mathrm{p}<0.0005$.

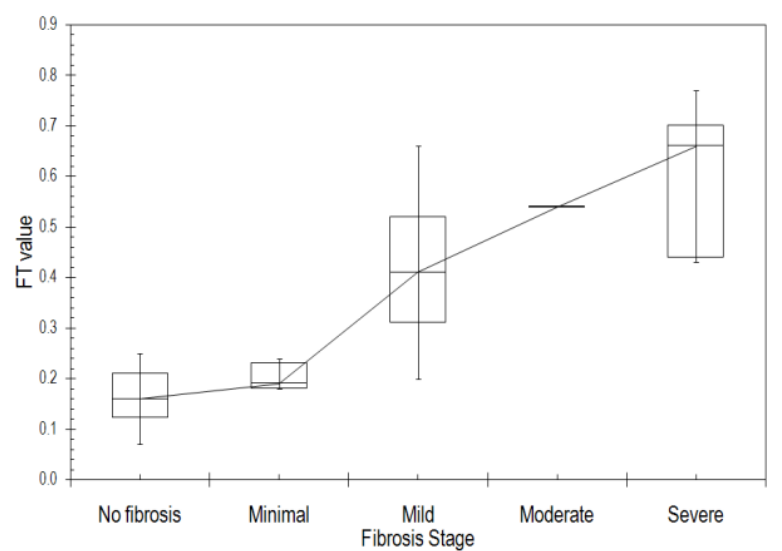

Fig. 1: Distribution of FT values as a function of hepatic fibrosis stage.

Patients were also classified according to the histological activity index (HAI) of their biopsies into 17 (34.0\%) with minimal activity (HIA 1-3), 25 (50.0\%) with mild activity (HIA 4-8) and 8 (16.0\%) with moderate activity (HIA 9-12).
In addition, the median $\left(25^{\text {th }}-75^{\text {th }}\right.$ percentile $)$ AT values increased linearly with advancing hepatic necroinflammatory activity grade. AT values were $0.19(0.12-$ $0.38)$ in patients with minimal activity, $0.25(0.12-0.48)$ in mild activity, and $0.72(0.56-0.90)$ in cases of moderate activity. As shown in Figure 2, the linear trend was highly significant with $\mathrm{p}<0.0005$. A highly significant correlation was also found between individual AT values and the respective HIA, $r=0.591, p<0.0005$.

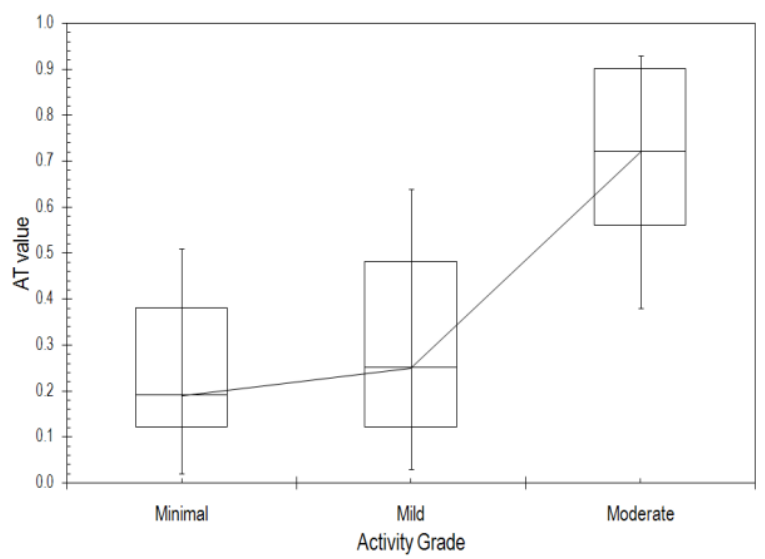

Fig. 2: Distribution of AT values as a function of hepatic activity grade.

A ROC curve was constructed (Fig.3) to demonstrate the ability of FT to diagnose patients with even a mild stage of fibrosis; discriminating them from those with no (or minimal) fibrosis. At FT cutoff $=0.25$, sensitivity was 92.3\% (two fibrosis cases missed) and specificity was $95.8 \%$ (one case falsely diagnosed). The overall accuracy was $94.0 \%$. A higher cutoff $(\mathrm{FT}=0.54)$ can be used to diagnose significant fibrosis (i.e., moderate or severe stages). As shown in Figure 3, this cutoff was associated with $71.4 \%$ sensitivity, $90.7 \%$ specificity and $88.0 \%$ accuracy.

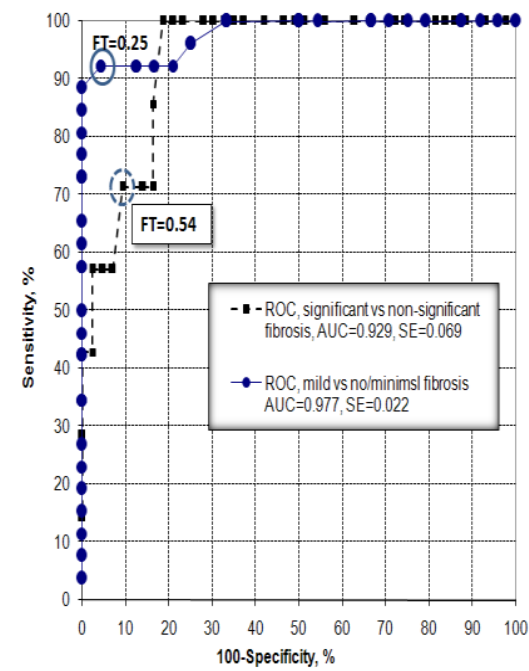

Fig. 3: ROC curves for the performance of FT in staging hepatic fibrosis. 


\section{Mortada El-Shabrawi, et al}

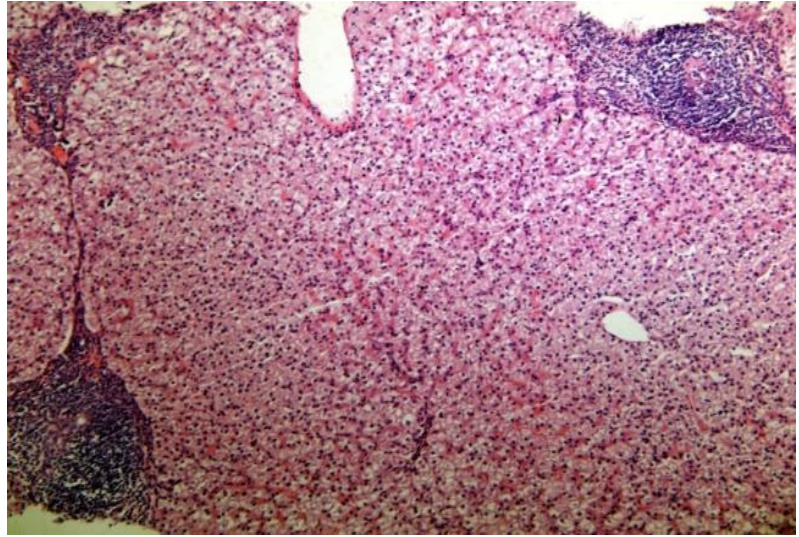

Fig. 4: Nine year old boy with chronic HCV infection

Fig 4 is an example of concordant liver biopsy which showed minimal fibrosis and mild necro-inflammatory activity in both biopsy blinded interpretation and FT/AT.

\section{Discussion:}

The availability of a noninvasive method for assessing hepatic fibrosis and activity is an undeniable advance in the management of patients with chronic HCV infection. Fibrotest and Actitest are currently available for routine use in adult patients. These tests have been validated in several studies and are an alternative to the gold standard method, liver biopsy. Because of the hospital stay required and the associated risks of the procedure, liver biopsy is becoming less and less acceptable for patients who increasingly demand a noninvasive method. New guidelines recommended use of the biochemical FT/AT for the initial work-up of $\mathrm{HCV}$ infection in routine practice (Fontanges et al., 2008).

To the best of our knowledge, no previous attempts to study the role of FT and AT in relation to liver biopsy in pediatric chronic $\mathrm{HCV}$ patients was carried out. The published work involved the role of FT and AT in adults and the role of biopsy in pediatric chronic HCV patients. In this pilot study, we attempted to verify the possibility of implementation of these noninvasive methods in pediatric chronic $\mathrm{HCV}$ patients, in whom they might be even more particularly needed.

Forty percent of our cases showed no fibrosis at biopsy, $8 \%$ minimal fibrosis, $38 \%$ mild fibrosis, $2 \%$ moderate fibrosis and $12 \%$ severe fibrosis. El-Hawary et al., (2007) and Guido et al., (2003) reported similar proportions of fibrosis in their HCV infected children. Although we noticed that there was a steady increase in age with increasing fibrosis, the difference did not reach statistical significance. Similar results were reported by Marcellin et al., (2002) where age was not a statistically significant feature associated with progression of fibrosis in patients with chronic HCV. Alternatively, some studies reported age to be an important factor in prediction of fibrosis (Habib et al., 2001). In our study, a highly significant linear trend was found between FT and the fibrosis stage with a highly statistically significant correlation also found.
In our patients, FT could successfully discriminate between patients with no fibrosis and those with mild, moderate and severe fibrosis (F1, F2 and F3 respectively), at a cut off value of 0.25 , with a sensitivity of $92.3 \%$ and a specificity of $95.8 \%$ and an overall accuracy of $94.0 \%$. Also FT successfully discriminated between patients with F1 and those with advanced fibrosis stages (F2-F3), at a cut off value of 0.54 , but with $71.4 \%$ sensitivity and $90.7 \%$ specificity and $88.0 \%$ overall accuracy.

The histological activity index (HIA) of our biopsies revealed $34 \%$ with minimal activity, $50 \%$ mild activity, $16 \%$ moderate activity with no severe activity found between the studied groups. The linear trend between AT related HAI was highly significant, with a significant correlation found between both parameters. Similarly, AT could successfully discriminate between patients with mild activity and those with moderate activity, at a cut off value of 0.54 . Patients with moderate activity could be predicted with a sensitivity of $87.5 \%$ and a specificity of $90.5 \%$ and an overall accuracy of $90.0 \%$. On the other hand, AT was less successful in discriminating between patients with mild activity and those with no (or almost no) activity, at a cut off value of 0.21 , with only $70 \%$ sensitivity and $59 \%$ specificity and $66 \%$ overall accuracy. Fontanges et al (2008) found that the distribution of the biochemistry activity (AT) and fibrosis indexes (FT) paralleled the distribution of the histological activity and fibrosis results respectively in 96 adult chronic $\mathrm{HCV}$ patients. For the activity score, at the best cut off value of 0.495 for A0-A1 versus A2-A3, the sensitivity was $70 \%$ and specificity $61 \%$. While for fibrosis, at the best cut off value of 0.385 the sensitivity and specificity were $74 \%$ and $61 \%$ respectively. Poynard et al., (2008) also, found a significant association between FT and fibrosis stage in liver biopsy. Both had similar accuracy with a trend in favor of FT. The specificity and sensitivity of FT were $83 \%$ and $62 \%$ respectively.

Poynard et al., (2000) observed that fibrosis progression was not normally distributed. The distribution suggested at least 3 populations: "rapid fibrosers", "intermediate fibrosers" and "slow fibrosers. The potential factors of FT variability are: normal transaminases ALT cause of liver disease and ethnic origin (Poynard et al., 2008). Discordance between FT and biopsy may be related to biopsy failure due mainly to sampling errors and to falsepositive and false-negative FT results related to Gilbert syndrome, hemolysis, or acute inflammation including specificity and detection limit for fibrosis, with $<5 \%$ false positives or false negatives (Poynard et al, 2004). Hence, these were among the exclusion criteria when selecting our patients. Yet, clinicians must interpret FT and AT with caution in patients with a significant elevation of ALT and/or GGT and/or alpha 2 macroglobulin which could overestimate hepatic injury (Fontanges et al., 2008).

FibroTest was a better predictor than biopsy staging for HCV complications; and was also a better predictor for $\mathrm{HCV}$ deaths in the study by Ngo et al (2006). FT was also indicative of response to virologic treatment and 


\section{Non Invasive Assessment of Hepatic Fibrosis.......}

permitted identification of patients with a high mortality risk. Biomarkers have shown error rates similar to or lower than those of small liver biopsies. Thus, they concluded that FT has prognostic values at least similar to that of liver biopsy, with the advantage of being noninvasive. On the other hand the role of the liver biopsy in chronic hepatitis C according to El-Hawary et al., (2007) would seem, at first, to be unquestioned as it provides much information in such a small package including confirmation of the diagnosis, exclusion of other liver diseases, and assessment of the grade and stage of the disease. They also added that the usefulness of the liver biopsy has received the endorsement of several national and international consensus conferences on the diagnosis and treatment of hepatitis C. Yet, liver biopsy is an invasive procedure, unpleasant for the patient, expensive, not free from severe complications, and prone to sampling errors due to the very small size of needle biopsy specimens (Bosch, 2009).

The benefit of FT also extends beyond chronic HCV infection. A meta-analysis by Poynard et al (2007) demonstrated that the diagnostic value of FT was similar in the four most frequent chronic liver diseases (HCV, HBV, NAFLD and ALD). It also demonstrated that the diagnostic value of FT, as for liver biopsy, was similar between all the adjacent fibrosis stages but without a specific "gray zone" or "inaccurate zone" between intermediate stages. FT, like biopsy, has lower diagnostic value to discriminate between two adjacent stages than between two extreme stages [17] (Ratziu et al.,2006). In addition, Ngo et al., (2008) found that FT has significant prognostic values at 4 years in patients infected by HBV, similar to that of liver biopsy.

The decision to treat (or not) in children with chronic $\mathrm{HCV}$ infection is currently a contentious endeavor (Narkewicz et al., 2007). A slowly progressive course this infection in children would favor deferred treatment, given that the multiple side effects of the currently available therapy observed in adults might be compounded by growth retardation in children. On the other hand, if pediatric trials show that children tolerate anti-viral therapy well with high sustained response rates, early treatment would be preferred over the potential for serious liver disease decades later. Mohan et al., (2007) from natural history study as well as other pediatric studies suggested that treatment can be deferred to adolescence or later as long as patients are followed closely. Therefore, in this pilot study we provoked the possibility of using the noninvasive biomarkers in children with chronic HCV infection aiming they would proof benefit in the follow up of these children who are exposed to longer duration of the disease. Thus, they will need the repetition of the tests for their close follow up in order to detect the appropriate time to begin therapy and in whom the repetition of the biopsy procedure would be most inconvenient.

Finally, FibroTest has been claimed to aid in the diagnosis of large esophageal varices and may therefore reduce the indication of endoscopic screening in cirrhotic patients (Thabut et al., 2006). Also, Fibrotest is correlated with the presence and degree of portal hypertension (Thabut et al., 2007).

Conclusion:

FT and AT are potential, reliable noninvasive markers for detection of fibrosis and necroinflammatory activity in pediatric patients with chronic HCV infection. Further studies are needed for their validation to avoid the need of repeated biopsies in these pediatric patients which could then be easily followed up by repeated FT and AT. Thus, detecting the appropriate time for initiating therapy would be more précised.

References:

1. Paik SW Goals of treatment, indication, and treatment for chronic hepatitis C] Korean J Gastroenterol. 2008 Jun;51(6):368-71

2. El-Hawary MA, El-Raziky MS, Esmat G, Soliman H, Abouzied A, El-Raziky M, El-Akel W, El-Sayed R, Shebl F, Shaheen AA, El-Karaksy H. Assessment of hepatic fibrosis in pediatric cases with hepatitis $\mathbf{C}$ virus in Egypt. World J Gastroenterol 2007; 13(20): 2846-2851

3. Medhat A, Shehata M, Magder LS, Mikhail N, AbdelBaki L, Nafeh M, Abdel-Hamid M,Strickland GT, Fix AD, . Hepatitis $c$ in a community in Upper Egypt: risk factors for infection. Am J Trop Med Hyg 2002; 66: 633-638

4. Habib M, Mohamed MK, Abdel-Aziz F, Magder LS, Abdel-Hamid M, Gamil F, Madkour S, Mikhail NN, Anwar W, Strickland GT, Fix AD, Sallam I. Hepatitis $C$ virus infection in a community in the Nile Delta: risk factors for seropositivity. Hepatology 2001; 33:248-253

5. Jonas MM Children with hepatitis C. Hepatology. 2002 Nov; 36(5 Suppl 1):S173-8

6. Wasley A and Alter MJ Epidemiology of hepatitis C; Geographic differences and temporal trends. Semin Liver Dis.2000; 20(1),1-16

7. Bosch J. Towards the non-invasive diagnosis of cirrhosis: The nuts-cirrhosis connection. Journal of Hepatology 50 (2009) 4-6

8. Goodman ZD, Makhlouf HR, Liu L, Balistreri W, Gonzalez-Peralta RP, Haber B, Jonas MM, Mohan P, Molleston JP, Murray KF, Narkewicz MR, Rosenthal P, Smith LJ, Robuck PR, Schwarz KB. Pathology of chronic hepatitis $\mathrm{C}$ in children: liver biopsy findings in the Peds-C Trial. Hepatology. 2008 Aug;48(2):691-2; author reply 692 
9. Ngo $\mathrm{Y}$, Munteanu M, Messous D, Charlotte F, Imbert-Bismut F, Thabut D, Lebray P, Thibault V, Benhamou Y, Moussalli J, Ratziu V and Poynard T. A Prospective Analysis of the Prognostic Value of Biomarkers (FibroTest) in Patients with Chronic Hepatitis C, Clinical Chemistry a 2006 52: 18871896,

10. Poynard $T$, Morra $R$, Ingiliz $P$, Imbert-Bismut $F$, Thabut D, Messous D, Munteanu M, Massard J, Benhamou Y, Ratziu V. Biomarkers of liver fibrosis. Adv Clin Chem. 2008;46:131-60.

11. Dolmazashvili E, Zhamutashvili M, Svanidze $M$, Nizharadze $N$, Abutidze A. Fibroscan and fibrotest/fibromax to assess liver fibrosis/cirrhosis in patients with chronic HBV and HCV infection in georgia. Georgian Med News. 2008 Dec;(165):83-7.

12. Poynard T, Morra R, Halfon P, Castéra L, Ratziu V, Imbert-Bismut F, Naveau S, Thabut D, Lebrec D, Zoulim F, Bourliere M, Cacoub P, Messous D, Muntenau $M$ and Victor de Ledinghen

Meta-analyses of FibroTest diagnostic value in chronic liver disease BMC Gastroenterol. 2007; 7: 40.

13.Poynard T, Ingiliz $P$, Elkrief L, Munteanu $M$, Lebray P, Morra R, Messous D, Imbert-Bismut F, Roulot D, Benhamou Y, Thabut D, and Ratziu V. Concordance in a world without a gold standard: A new non-jnvasive methodology for improving accuracy of fibrosis markers PLoS ONE. 2008; 3(12): e3857.

14. Poynard T, Imbert-Bismut F, Munteanu M, Ratziu V: FibroTest-FibroSURE: towards a universal biomarker of liver fibrosis? Expert Rev Mol Diag 2005, 5:15-21.

15. Myers RP, Tainturier MH, Ratziu V, Piton A, Thibault V, Imbert-Bismut F, Messous D, Charlotte F, Di Martino V, Benhamou Y, Poynard T: Prediction of liver histological lesions with biochemical markers in patients with chronic hepatitis Hepatol 2003, 39:222-230.

16. Naveau S, Raynard B, Ratziu V, Abella A, ImbertBismut F, Messous D, Beuzen F, Capron F, Thabut D, Munteanu M, Chaput JC, Poynard T: Biomarkers for the prediction of liver fibrosis in patients with chronic alcoholic liver disease. Clin Gastroenterol Hepatol 2005, 3:167-174.
17. Ratziu V, Massard J, Charlotte F, Messous D, Imbert-Bismut F, Bonyhay L, Tahiri M, Munteanu M, Thabut D, Cadranel JF, Le Bail B, De Ledinghen V, Poynard T. The LIDO Study Group and the CYTOL Study Group: Diagnostic value of biochemical markers (FibroTest-FibroSURE) for the prediction of liver fibrosis in patients with non-alcoholic fatty liver disease. BMC Gastroenterology 2006, 6:6.

18. Rothman $\mathrm{KJ}$. Declaration of Helsinki should be strengthened British Medical Journal 2000 12; 321(7258): 442-445.

19. Poynard T. Diagnosis method of inflammatory, fibrotic, or cancerous disease using biochemical markers. US Patent 6631 330. October 7, 2003.

20. Myers RP, Benhamou Y, Imbert-Bismut F, Thibault V, Bochet $M$, Charlotte F, et al. Serum biochemical markers accurately predict liver fibrosis in HIV and hepatitis C virus-coinfected patients. AIDS 2003; 17:1-5.

21. Thabut D, Simon M, Myers RP, Messous D, Thibaut V, Imbert-Bismut F, et al. Non-invasive prediction of fibrosis in patients with chronic hepatitis $C$ [letter]. Hepatology 2003; 37:1220-1221.

22. Callewaert $\mathbf{N}$, Van Vlierberghe H, Van Hecke A, Laroy W, Delanghe J, Contreras R. Noninvasive diagnosis of liver cirrhosis using DNA sequencerbased total serum protein glycomics. Nat Med 2004; 10:1-6.

23. Castéra L, Vergniol J, Foucher J, Brigitte Le Bail B, Chanteloup E, Haaser M, et al. Prospective comparison of transient elastography, FibroTest, APRI and liver biopsy for the assessment of fibrosis in chronic hepatitis C. Gastroenterology 2005;128:343-350.

24. Halfon, Portal I, Renou C, et al. Independent prospective multicenter validation of biochemical markers (FibroTest-ActiTest) for the prediction of liver fibrosis and activity in patients with chronic hepatitis C. Am J Gastroenterol 2006;101:547-555.

25.Poynard $T$, Munteanu $M$, Imbert-Bismut F, Charlotte F, Thabut D, Le Calvez S, et al. Prospective analysis of discordant results between biochemical markers and biopsy in patients with chronic hepatitis C. Clin Chem 2004;50:1344-1355.

26.Bedossa P, Poynard T. An algorithm for the grading of activity in chronic hepatitis $\mathbf{C}$. The METAVIR Cooperative Study Group. Hepatology 1996;24:289-293. 


\section{Non Invasive Assessment of Hepatic Fibrosis.......}

27. Fontanges T, Bailly F, Trepo E, Chevallier $\mathbf{M}$, Maynard- Muet M, Nalet B, Beorchia S, Pillon D, Moindrot H, Froissart B, Siaoui M, Tinnel X, Pradat $P$, Trepo C. Discordance between biochemical of liver activity and fibrosis (Actitest-Fibrotest) and liver biopsy markers in patients with chronic hepatitis C. Gastroenterologie Clinique et Biologique 2008; 32: 858-865.

28. Guido M, Bortolotti F, Leandro G, Jara P, Hierro L, Larrauri J, Barbera C, Giacchino R, Zancan L, Balli F, Crivellaro C, Cristina E, Pucci A, Rugge M. Fibrosis in chronic hepatitis $C$ acquired in infancy: is it only a matter of time? Am J Gastroenterol 2003; 98: 660663

29. Marcellin P, Asselah T, Boyer N. Fibrosis and disease progression in hepatitis C. Hepatology 2002; 36: S47-S56

30. Poynard T, Ratziu V, Benmanov Y, Di Martino V, Bedossa P, Opolon P. Fibrosis in patients with chronic hepatitis $C$ : detection and significance. Semin Liver Dis 2000; 20: 47-55
31.Thabut D, Trabut JB, Massard J, Rudler M, Muntenau M, Messous D, Poynard T. Non-invasive diagnosis of large oesophageal varices with FibroTest in patients with cirrhosis: a preliminary retrospective study. Liver Int. 2006 Apr;26(3):2718.

32.Thabut D, Imbert-Bismut F, Cazals-Hatem D, Messous D, Muntenau M, Valla DC, Moreau R, Poynard T, Lebrec D. Relationship between the Fibrotest and portal hypertension in patients with liver diseaseAliment Pharmacol Ther. 2007 Aug 1;26(3):359-68.

33. Ngo Y, Yves Benhamou, Thibault V, Ingiliz P, Muntenau M, Lebray P, Thabut D, Morra R, Messous D, Charlotte F, Imbert-Bismut F, RousselotBonnefont D, Moussalli J, Ratziu V, and Poynard T. An Accurate Definition of the Status of Inactive Hepatitis B Virus Carrier by a Combination of Biomarkers (FibroTest-ActiTest) and Viral Load. PLoS ONE. 2008; 3(7): e2573

34 Narkewicz MR, Cabrera R, González-Peralta RP. The "C" of viral hepatitis in children. Semin Liver Dis. 2007 Aug; 27(3):295-311 